\title{
Sleep and Synaptic Down-Selection
}

\author{
Giulio Tononi and Chiara Cirelli
}

\begin{abstract}
Sleep is universal, tightly regulated, and many cognitive functions are impaired if we do not sleep. But why? Why do our brains need to disconnect from the environment for hours every day? We discuss here the synaptic homeostasis hypothesis (SHY), which proposes that sleep is the price the brain pays for plasticity, to consolidate what we already learned, and be ready to learn new things the next day. In brief, new experiments show that the net strength of synapses increases with wake and decreases with sleep. As we discuss, these findings can explain why sleep is necessary for the well-being of neural cells and brain circuits, and how the regulation of synaptic strength may be a universal, essential function of sleep.
\end{abstract}

\section{Sleep Is Universal and Probably Essential, But Why?}

We spend on average a third of our time asleep, but the functions of sleep remain elusive (Mignot 2008; Siegel 2008). This is even more puzzling if one considers that overall, during sleep the brain is almost as active as in waking life: neurons fire at comparable rates as in wake, metabolism is only slightly reduced (Steriade and Hobson 1976). So if sleep is not simply a passive state, during which brain cells can rest, why does it disconnect from the environment, turns on spontaneous activity, experiences vivid dreams but forms no new memories? This question is all the more intriguing since sleep is universal (Cirelli and Tononi 2008). For example, even animals that cannot afford to sleep in the regular manner because they are constantly on the move, such as several species of cetaceans, have found a clever way of cheating with sleep. Thus dolphins continue to swim and breathe with one hemisphere, while the other half of their brain is deep asleep, showing EEG slow waves just as in other mammals (Oleksenko et al. 1992). Though nature offers some

\footnotetext{
G. Tononi $(\bowtie) \cdot$ C. Cirelli

Department of Psychiatry, University of Wisconsin-Madison, 6001 Research Park Blvd, Madison, WI 53719, USA

e-mail: gtononi@wisc.edu; ccirelli@wisc.edu 
remarkable case studies in sleep self-deprivation-some Alaskan sandpipers briefly forgo regular sleep during the intense mating season (Lesku et al. 2012), and migrating birds make do for a while on a third of their normal sleep (Rattenborg et al. 2004) - there is so far no positive proof of any species that lacks sleep completely (Cirelli and Tononi 2008). We now know that even invertebrates sleep, including fruit flies (Hendricks et al. 2000; Shaw et al. 2000), honeybees (Kaiser 1988), and octopus (Brown et al. 2006), though we still do not know how much spontaneous activity goes on in their sleeping brains. Furthermore, fruit flies not only sleep many hours a night but, just like mammals and birds, if they are kept awake, their sleep becomes longer and more intense-a process called sleep homeostasis (Huber et al. 2004b). Therefore, sleep does not seem to be a niche adaptation, like hibernation, which may benefit some species that need to save energy or avoid trouble when they have nothing better to do. Rather, sleep seems to serve some essential, universal function, akin to temperature regulation or digestion (Cirelli and Tononi 2008). What's more, it is a function that requires the brain to work off-line, despite the dangers of being temporarily disconnected from the environment. But what is this function?

\section{Sleep Helps Memory Consolidation, Gist Extraction, and Integration}

A popular idea that has gained much attention recently is that sleep may be important for memory. There are now plenty of experiments showing that, after a night of sleep and sometimes just after a nap, newly formed memories are preserved better than if one had spent the same amount of time awake. That is, sleep benefits memory consolidation (Rasch and Born 2013). This benefit is especially clear for declarative memories - those one can recollect consciously, such as lists of words or associations between pictures and places. But non-declarative memories, such as perceptual and motor skills, can also profit from sleep. For instance, if you try to reach a target on the screen with the mouse while, unbeknownst to you, the cursor is systematically rotated, you slowly learn to compensate for the rotation and get progressively better. If you sleep over it, you improve further, and your movements become smooth (Huber et al. 2004a). These experimental results fit the common observation that after intensive learning, say practicing a piece over and over on the guitar, performance often becomes fluid only after a night of sleep. It is likely that, when you learn by trial-and-error and repeatedly activate certain brain circuits, many synapses end up strengthening, not only when you play the right notes well, but also when you do it badly, or fumble other notes. The result is that, while by practicing you get better and better on average, your performance remains a bit noisy and variable. After sleep, it is as if the core of what you learned had been preserved, whereas the chaff is eliminated-that is, sleep seems to notch-up the signal-to-noise ratio (Rasch and Born 2013; Tononi and Cirelli 2014). Something 
similar may happen also with declarative memories: in the face of the hundreds of thousands of scenes we encounter in waking life, memory is particularly effective at gist extraction, where the details (the noise) may be lost, but the main point of what happened to you (the signal) is preserved. So far, it seems that the memory benefits of sleep, especially for declarative memories, are due primarily to non-rapid eye movement (NREM) sleep, but in some instances REM sleep or a combination of NREM-REM cycles may also play a role (Rasch and Born 2013). Of course, while sleep is undoubtedly important for memory consolidation, one should not forget that memories can also consolidate during wake. Moreover, to some extent sleep helps memory consolidation simply because it reduces the interference caused by later memory traces, since when you sleep you stop learning new things (Ellenbogen et al. 2006).

Another process that may benefit from sleep is the integration of new with old memories. Psychologists have long recognized that one tends to learn new material better if it has many points of contacts with previous knowledge. For example, a new word in a language you already know fairly well is easier to remember than a new word in a completely unknown language. This process of integration certainly occurs during wake- a memorable stimulus will activate, consciously or subconsciously, a vast network of associations throughout the brain (read synapses), with which it may become linked. However, sleep may be a particularly good time to assess which of the new memories fit better and which worse with the vast amount of organized older memories - also known as schemata - that are stored in brain circuits (Lewis and Durrant 2011). This is because during sleep it is possible to activate a large number of circuits in many different combinations without worrying about the consequences for behavior, something that is not advisable during wake, when one must stick to the situation at hand. For example, in real life it would not be a good idea to reminisce about your father's old car being of a similar color as that of the large truck that is rapidly approaching. In a dream, instead, it is perfectly fine to put your father in the truck's driver seat, realize later that it is actually a school bus, and notice that it is filled with old people who resemble your colleagues. Perhaps during sleep your brain is sifting through old memories and trying out which new ones fit best overall, while getting rid of the rest, just as it does with gist extraction.

\section{Sleep Helps Memory, But How? Is It Replay or Synaptic Down-Selection?}

The ongoing activity in the brain throughout sleep, then, could have something to do with consolidating memory traces, extracting their gist, and integrating new with old memories (Born et al. 2006; Rasch and Born 2013). This idea is supported by studies performed over the past 20 years, first in rodents and then in primates, which show that patterns of neural activity during sleep often resemble those recorded 
during wake (Wilson and McNaughton 1994; Kudrimoti et al. 1999; Nadasdy et al. 1999; Hoffman and McNaughton 2002). For example, when a rat learns to navigate a maze, different hippocampal neurons fire in different places, in specific sequences. Presumably, each sequence is encoded in memory by strengthening the connections between neurons firing one after the other. During subsequent sleep, especially NREM sleep, these sequences are "replayed" above chance (though neither very often nor very faithfully). Based on this evidence, many researchers think that sleep "replay" may consolidate memories by further reinforcing the synaptic connections that had been strengthened in wake, leading to synaptic consolidation. There may also be some system-level consolidation, based on evidence that over time memories may be shuttled around in the brain. For example, the hippocampus may provide early storage, after which memories are transferred to connected cortical areas, and sleep may help this transfer (Girardeau et al. 2009). However, there is also evidence that the "replay" of neural circuits can also occur in wake, not just in sleep (Karlsson and Frank 2009), and "preplay" can also occur during wake before learning (Dragoi and Tonegawa 2011).

An interesting alternative is that sleep may be a time not so much for rehearsal, but for down-selection (Tononi and Cirelli 2014). In essence, the idea is this: when the brain sleeps, spontaneous neuronal firing activates many circuits in many different combinations, both new memory traces, which may be particularly prone to reactivation, and old networks of associations. But instead of strengthening whatever synapses are activated the most, which would lead to learning things that never happened, the brain could reverse plasticity rules, and promote the activitydependent weakening of connections. Indeed, an efficient way to do so would be to implement a selectional, competitive process. For example, synapses that are reactivated most strongly and consistently during sleep would be protected and survive mostly unscathed, whereas synapses that are comparatively less activated would be depressed. This down-selection process would literally ensure the survival of those circuits that are "fittest," either because they were strengthened repeatedly during wake (the signal, i.e., the right notes on the guitar) or because they are better integrated with previous, older memories (a new word in a known language). Instead, synapses involved in circuits that were only occasionally strengthened during wake (the noise, i.e., fumbled notes on the guitar), or fit less with old memories (a new word in an unknown language), would be depressed and possibly eliminated. In this way, synaptic down-selection during sleep would promote memory consolidation by increasing signal-to-noise ratios, thereby favoring gist extraction and the integration of new memories with established knowledge. As an additional bonus, down-selection would also make room for another cycle of synaptic strengthening during wake. Indeed, there are several indications that sleep, in addition to memory consolidation, gist extraction, and integration, is particularly beneficial to memory acquisition: quite a few studies have shown after a night of sleep you can learn new material much better than after having been awake all day.

Finally, down-selection based on the systematic reactivation of neural circuits old and new would also explain why prolonged quiescence and disconnection from 
the environment are important — that is, why one needs to be asleep. This is because sleep is the perfect time for the brain to try out many different scenarios without worrying about behaving appropriately in the real world. Only in this way can the brain go through a large repertoire of situations, collect fair statistics about how each synapse is activated in the context of the entire set of stored memories (how well it fits), and reorganize its networks accordingly. Otherwise, the synapses that were strengthened most recently would always be favored (say you spent the entire day trying out the guitar) at the expense of others that are equally important (you also know how to type, and you would not want to forget it), irrespective of how the new memories fit with your previous knowledge.

In the end, cycles of net strengthening of connections during wake followed by net weakening during sleep may constitute an excellent selectional strategy that implements a healthy reality check: neural activity patterns triggered during wake, when the brain is connected with the environment, would tend to be reinforced, whereas activation patterns triggered during sleep, when the brain is disconnected from the environment and makes up its own imaginary scenarios, would be weeded out systematically.

\section{Synapses Get Stronger in Wake and Weaker in Sleep}

There is converging evidence for synaptic down-selection during sleep (Tononi and Cirelli 2014). Experiments performed in fruit flies, rodents, and humans, all seem to indicate that the strength of connections among neurons increases during wake and decreases during sleep. For example, when fruit flies spend the day in an environment with plenty of opportunity for interactions with other flies (a "fly mall"), by evening time there are almost $70 \%$ more synaptic spines-the little protrusion where an incoming axon makes contact with a dendrite-than there were in the morning, and this is true throughout their brain. The next morning the number of spines goes back to baseline, but only if flies are allowed to sleep (Bushey et al. 2011). In adolescent mice one sees a similar phenomenon: in the cerebral cortex the number of synaptic spines tends to grow during wake and to decrease during sleep, although the changes are smaller than in flies (Maret et al. 2011; Yang and Gan 2011). In adult rodents it is not the number of synaptic spines that changes with wake and sleep, but their strength. This is indicated by an increase in the number of AMPA receptors in the synapses after wake, and a decrease after sleep (Vyazovskiy et al. 2008). AMPA receptors are responsible for the bulk of excitatory neurotransmission in mammalian brains, and the potentiation or depression of synapses is ultimately achieved by increasing or decreasing their number. Other experiments have shown that, if one stimulates electrically neural fibers in the cortex, the response one gets from the target neurons is larger after a few hours of wake, and smaller after sleep, and we know that these responses are usually larger when synapses are strong, and smaller when they are weak (Vyazovskiy et al. 2008). A similar experiment was performed in humans using transcranial 
magnetic stimulation - a short magnetic pulse applied to the scalp to activate the underlying neurons - and high-density EEG to record the strength of the responses of the rest of the cerebral cortex. The results were clear: the longer the subject was awake, the larger the responses, and it took a night of sleep for the responses to return to baseline (Huber et al. 2013).

One should emphasize that exactly how this down-selection process would take place remains unclear, and the account above remains speculative. Indeed, the precise mechanisms are likely to vary in different species, in different brain structures, and in different developmental periods. For example, it is not known whether in invertebrates sleep is accompanied by intense neuronal activation or not-perhaps there the weakening of synapses can be accomplished without having to go through a large repertoire of old memories. Similarly, it may be that NREM sleep is the ideal time for weakening synapses in an activity-dependent manner in the cerebral cortex, due to the occurrence of slow waves; but that in the hippocampus, which does not generate slow waves, down-selection may happen preferentially during the faster, theta waves of REM sleep (Grosmark et al. 2012). Irrespective of the specific mechanisms, the evidence is strong, in several species, that overall synaptic strength goes up during wake and down during sleep. And if this is so, it has implications concerning the role of sleep that go beyond its benefits to memory consolidation and integration, as we will now briefly discuss.

\section{SHY: The Price of Plasticity}

Perform a simple experiment: before you go to bed, try and remember as many things as you can that happened to you today. If you are serious and systematic about it, starting with your first thought upon awakening, the first thing you did, what you had for breakfast, where you had breakfast, and so on, the list will be very long, and very boring. Now even this list would be very incomplete. If you were to wear a camera on your head recording all that happened to you, and if we were to then show you snapshots from the recordings, you would suddenly recognize many other things that happened to you that you did not initially recollect. And then there are perceptual and motor skills that you have acquired or refined during the day, such as the guitar piece you practiced. Obviously, over a typical day a lot of things must have left a trace in your brain. We still do not know what proportion of the trillions of synapses in your brain is actually changed by a day of wake: is it 0.01 , $1,10 \%$ or even more? But for sure a lot of synapses must have been strengthened, as suggested not only by your little evening thought experiment, but also by the experimental evidence reviewed in the previous section.

Now the crucial thing to realize is that all this learning, if it is reflected in the strengthening of synapses, does not come for free. First of all, stronger synapses consume more energy. For its weight, the brain is by far the most expensive organ of the body - accounting for almost $20 \%$ of the energy budget — and of that budget, two thirds or more is for supporting synaptic activity. So if we learn by 
strengthening synapses, one could say that we wake up with an efficient engine and we end the day with a gas-guzzler. Also, a net strengthening of synapses is a major source of cellular stress, due to the need to synthesize and deliver cellular constituents ranging from mitochondria to synaptic vesicles to various proteins and lipids. Clearly, learning by strengthening synapses cannot go on indefinitely-day after day-and something must be done about it. That something, says the synaptic homeostasis hypothesis, also known as SHY, is the down-selection of synapses down to a baseline level that is sustainable both in terms of energy consumption and cellular stress. And that, says SHY, is the essential function of sleep. In short, sleep is the price we pay for being able to learn and adapt to novel environments when we are awake-most generally, it is the price we pay for plasticity. If this is indeed the essential function of sleep, it is only fitting that, as sleep-dependent synaptic downselection relieves neural cells of the metabolic burdens accumulated during wake in the service of plasticity, it does so in a smart way, all along benefitting memory consolidation and integration, while also resetting the conditions for efficiently acquiring new memories when we wake up. This would not be the first time that evolution catches many birds with one stone.

Acknowledgements Supported by NIMH grant R01MH099231 to CC and GT, and NINDS grant P01NS083514 to CC and GT.

Open Access This chapter is distributed under the terms of the Creative Commons AttributionNoncommercial 2.5 License (http://creativecommons.org/licenses/by-nc/2.5/) which permits any noncommercial use, distribution, and reproduction in any medium, provided the original author(s) and source are credited.

The images or other third party material in this chapter are included in the work's Creative Commons license, unless indicated otherwise in the credit line; if such material is not included in the work's Creative Commons license and the respective action is not permitted by statutory regulation, users will need to obtain permission from the license holder to duplicate, adapt or reproduce the material.

\section{References}

Born J, Rasch B, Gais S (2006) Sleep to remember. Neuroscientist 12:410-424

Brown ER, Piscopo S, De Stefano R, Giuditta A (2006) Brain and behavioural evidence for restactivity cycles in Octopus vulgaris. Behav Brain Res 172:355-359

Bushey D, Tononi G, Cirelli C (2011) Sleep and synaptic homeostasis: structural evidence in Drosophila. Science 332:1576-1581

Cirelli C, Tononi G (2008) Is sleep essential? PLoS Biol 6:e216

Dragoi G, Tonegawa S (2011) Preplay of future place cell sequences by hippocampal cellular assemblies. Nature 469:397-401

Ellenbogen JM, Hulbert JC, Stickgold R, Dinges DF, Thompson-Schill SL (2006) Interfering with theories of sleep and memory: sleep, declarative memory, and associative interference. Curr Biol 16:1290-1294

Girardeau G, Benchenane K, Wiener SI, Buzsaki G, Zugaro MB (2009) Selective suppression of hippocampal ripples impairs spatial memory. Nat Neurosci 12:1222-1223 
Grosmark AD, Mizuseki K, Pastalkova E, Diba K, Buzsaki G (2012) REM sleep reorganizes hippocampal excitability. Neuron 75:1001-1007

Hendricks JC, Finn SM, Panckeri KA, Chavkin J, Williams JA, Sehgal A, Pack AI (2000) Rest in Drosophila is a sleep-like state. Neuron 25:129-138

Hoffman KL, McNaughton BL (2002) Coordinated reactivation of distributed memory traces in primate neocortex. Science 297:2070-2073

Huber R, Ghilardi MF, Massimini M, Tononi G (2004a) Local sleep and learning. Nature 430:78-81

Huber R, Hill SL, Holladay C, Biesiadecki M, Tononi G, Cirelli C (2004b) Sleep homeostasis in Drosophila melanogaster. Sleep 27:628-639

Huber R, Maki H, Rosanova M, Casarotto S, Canali P, Casali AG, Tononi G, Massimini M (2013) Human cortical excitability increases with time awake. Cereb Cortex 23:332-338

Kaiser W (1988) Busy bees need rest, too. Behavioral and electromyographic sleep signs in honeybees. J Comp Physiol A 163:565-584

Karlsson MP, Frank LM (2009) Awake replay of remote experiences in the hippocampus. Nat Neurosci 12:913-918

Kudrimoti HS, Barnes CA, McNaughton BL (1999) Reactivation of hippocampal cell assemblies: effects of behavioral state, experience, and EEG dynamics. J Neurosci 19:4090-4101

Lesku JA, Rattenborg NC, Valcu M, Vyssotski AL, Kuhn S, Kuemmeth F, Heidrich W, Kempenaers B (2012) Adaptive sleep loss in polygynous pectoral sandpipers. Science 337:1654-1658

Lewis PA, Durrant SJ (2011) Overlapping memory replay during sleep builds cognitive schemata. Trends Cogn Sci 15:343-351

Maret S, Faraguna U, Nelson A, Cirelli C, Tononi G (2011) Sleep and wake modulate spine turnover in the adolescent mouse cortex. Nat Neurosci 14:1418-1420

Mignot E (2008) Why we sleep: the temporal organization of recovery. PLoS Biol 6:e106

Nadasdy Z, Hirase H, Czurko A, Csicsvari J, Buzsaki G (1999) Replay and time compression of recurring spike sequences in the hippocampus. J Neurosci 19:9497-9507

Oleksenko AI, Mukhametov LM, Polyakova IG, Supin AY, Kovalzon VM (1992) Unihemispheric sleep deprivation in bottlenose dolphins. J Sleep Res 1:40-44

Rasch B, Born J (2013) About sleep's role in memory. Physiol Rev 93:681-766

Rattenborg NC, Mandt BH, Obermeyer WH, Winsauer PJ, Huber R, Wikelski M, Benca RM (2004) Migratory sleeplessness in the white-crowned sparrow (Zonotrichia leucophrys gambelii). PLoS Biol 2:E212

Shaw PJ, Cirelli C, Greenspan RJ, Tononi G (2000) Correlates of sleep and waking in Drosophila melanogaster. Science 287:1834-1837

Siegel JM (2008) Do all animals sleep? Trends Neurosci 31:208-213

Steriade M, Hobson J (1976) Neuronal activity during the sleep-waking cycle. Prog Neurobiol 6:155-376

Tononi G, Cirelli C (2014) Sleep and the price of plasticity: from synaptic and cellular homeostasis to memory consolidation and integration. Neuron 81:12-34

Vyazovskiy VV, Cirelli C, Pfister-Genskow M, Faraguna U, Tononi G (2008) Molecular and electrophysiological evidence for net synaptic potentiation in wake and depression in sleep. Nat Neurosci 11:200-208

Wilson MA, McNaughton BL (1994) Reactivation of hippocampal ensemble memories during sleep. Science 265:676-679

Yang G, Gan WB (2011) Sleep contributes to dendritic spine formation and elimination in the developing mouse somatosensory cortex. Dev Neurobiol 72:1391-1398 